QUARTERLY OF APPLIED MATHEMATICS

VOLUME LXV, NUMBER 1

MARCH 2007, PAGES 145-153

S $0033-569 X(06) 01032-9$

Article electronically published on December 14, 2006

\title{
A CONSTRAINT VARIATIONAL PROBLEM ARISING IN STELLAR DYNAMICS
}

\author{
$\mathrm{BY}$ \\ MAHIR HADZIC \\ Division of Applied Mathematics, Brown University, Providence, Rhode Island 02912
}

\begin{abstract}
We use the compactness result of A. Burchard and Y. Guo to analyze the reduced 'energy' functional arising naturally in the stability analysis of steady states of the Vlasov-Poisson system (cf. Sánchez and Soler, to appear, and Hadžić, 2005). We consider the associated variational problem and present a new proof that puts it in the general framework for tackling the variational problems of this type, given by Y. Guo and G. Rein (cf. Rein, 2005 and Rein, 2002).
\end{abstract}

1. Introduction and statement of the result. Our starting point is the VlasovPoisson system

$$
\begin{gathered}
\partial_{t} f+v \cdot \nabla_{x} f-\nabla_{x} U \cdot \nabla_{v} f=0, \\
\Delta U=4 \pi \rho, \quad \lim _{|x| \rightarrow \infty} U(t, x)=0, \\
\rho(t, x)=\int f(t, x, v) d v,
\end{gathered}
$$

where the dynamic variable $f=f(t, x, v)$ is the number density of a large ensemble of particles which interact by the gravitational potential $U=U(t, x)$. The variables $x$, $v \in \mathbb{R}^{3}$ denote position and velocity, $t \in \mathbb{R}$ is the time variable, and $\rho=\rho(t, x)$ is the spatial mass density induced by $f$. This system models a galaxy (stars take the role of particles).

Questions of nonlinear stability of stationary solutions to the Vlasov-Poisson system initiated many developments in recent years ( cf. 5 for a self-contained overview). The core idea was to recognize that a whole class of polytropic steady states can be obtained as minimizers of so-called energy-Casimir functionals. Once this connection is established, one makes use of the minimization property of steady states to deduce their non-linear

Received April, 2006.

2000 Mathematics Subject Classification. Primary 49K20.

This paper is a part of the author's Diploma thesis. I would like to thank Gerhard Rein for introducing me to the problem and for many stimulating discussions.

E-mail address: hadzic@dam.brown.edu

(C)2006 Brown University Reverts to public domain 28 years from publication 
stability. We introduce the notation

$$
L_{+}^{p}\left(\mathbb{R}^{n}, M\right):=\left\{f \in L^{p}\left(\mathbb{R}^{n}\right) ; f \geq 0 \text { a.e., }\|f\|_{L^{p}\left(\mathbb{R}^{n}\right)}=M\right\},
$$

and define kinetic and potential energy

$$
\begin{gathered}
E_{\text {kin }}(f):=\frac{1}{2} \iint|v|^{2} f(x, v) d v d x \\
E_{\mathrm{pot}}(f):=-\frac{1}{8 \pi} \int\left|\nabla U_{f}(x)\right|^{2} d x=-\frac{1}{2} \iint \frac{\rho_{f}(x) \rho_{f}(y)}{|x-y|} d x d y,
\end{gathered}
$$

where $\rho_{f}(x)=\int f(x, v) d v$. It is well known that the total energy

$$
E(f):=E_{\text {kin }}(f)+E_{\text {pot }}(f)
$$

is conserved along the solutions of the Vlasov-Poisson system (1.1)-(1.3). By abuse of notation we shall also write

$$
E_{\mathrm{pot}}(\rho)=-\frac{1}{2} \iint \frac{\rho(x) \rho(y)}{|x-y|} d x d y .
$$

The polytropic solutions are solutions of the form

$$
f_{\mu}(x, v):=\left(E_{0}-|v|^{2} / 2-U(|x|)\right)_{+}^{\mu},
$$

where $(f)_{+}$denotes the positive part of the function $f, E_{0} \in \mathbb{R}$ is a constant and $-1 / 2<$ $\mu<7 / 2$. For a certain range of $\mu$ the polytropes with prescribed mass $M$ were shown to be minimizers of the energy-Casimir functional

$$
E_{C}(f)=E(f)+\int Q(f(x, v)) d x d v
$$

under the constraint $f \in L_{+}^{1}\left(\mathbb{R}^{6}, M\right)$. By formulating the problem in terms of spatial densities $\rho=\int f(., v) d v$ in [6], the author naturally reduced it to the problem of minimizing a functional of the form

$$
E_{C}^{r}(\rho)=\int \Phi(\rho(x)) d x+E_{\text {pot }}(\rho)
$$

under the constraint $\rho \in L_{+}^{1}\left(\mathbb{R}^{3}, M\right)$. The notion of reduction and the exact relations between $Q$ and $\Phi$ are carefully analyzed in [6], where a concentration-compactness type argument is used to deal with the variational problem. A. Burchard and Y. Guo showed that it suffices to restrict the minimization procedure to the set of symmetrically decreasing functions $\rho$ (cf. [1, Thm. 1]). This makes the solution of the reduced variational problem simpler. In the review paper [5] this technique is put in a formal framework involving several steps, indicating the possible genericity of this approach. In [7, Ó. Sánchez and J. Soler approach the stability question by regarding the problem of minimizing the energy $E(f)$ over the set of positive functions with prescribed $L^{1}$ and $L^{1+1 / \mu}$ norms, with $\mu \in] 0,7 / 2[$. More precisely, they minimize the functional $E$ over the constraint set

$$
\Gamma_{M, J}^{\mu}:=L_{+}^{1}\left(\mathbb{R}^{6}, M\right) \cap L_{+}^{1+1 / \mu}\left(\mathbb{R}^{6}, J\right) .
$$

We denote

$$
I_{M, J}^{\mu}:=\inf \left\{E(f) ; f \in \Gamma_{M, J}^{\mu}\right\} .
$$


The crucial difference to the method used by Y. Guo and G. Rein is that in this case we have to deal with two simultaneous constraints. The crux of the method is to reduce the energy functional to a functional defined only over spatial densities $\rho$ and at the same time to keep only one constraint in the minimization procedure. The new equivalent problem, derived in [7], is to minimize

$$
E_{J}^{\mu}(\rho):=\frac{K_{1,1}}{J^{\frac{2(\mu+1)}{3}}}\left(\int \rho^{\frac{2 \mu+5}{2 \mu+3}} d x\right)^{\frac{2 \mu+3}{3}}+E_{\mathrm{pot}}(\rho)
$$

over the constraint set

$$
F_{M}^{\mu}:=L_{+}^{1}\left(\mathbb{R}^{3}, M\right) \cap L^{\frac{2 \mu+5}{2 \mu+3}}\left(\mathbb{R}^{3}\right) .
$$

$K_{1,1}$ is just a constant arising from the reduction procedure, and its value does not play a role for the rest of the paper. For details, see [7. We denote

$$
R_{M, J}^{\mu}:=\inf \left\{E_{J}^{\mu}(\rho) ; \rho \in F_{M}^{\mu}\right\}
$$

and

$$
\Psi(\rho)=\int \rho^{\frac{2 \mu+5}{2 \mu+3}} d x, \quad K:=\frac{K_{1,1}}{J^{\frac{2(\mu+1)}{3}}} .
$$

The above mentioned equivalence holds in the following sense:

LEmma 1.1 (Equivalence of the variational principles). The variational problems of minimizing $E$ over the constraint set $\Gamma_{M, J}^{\mu}$ and $E_{J}^{\mu}$ over the constraint set $F_{M}^{\mu}$, are equivalent in the following sense:

(1) The infima $I_{M, J}^{\mu}$ and $R_{M, J}^{\mu}$ (cf. (1.5) and (1.6) respectively) coincide, i.e. $I_{M, J}^{\mu}=$ $R_{M, J}^{\mu}$

(2) If $\left(f_{n}(\cdot, \cdot)\right) \subset \Gamma_{M, J}^{\mu}$ is a minimizing sequence of the functional $E$, then the sequence $\left(\rho_{n}(\cdot)\right)=\left(\int f_{n}(\cdot, v) d v\right) \subset F_{M}^{\mu}$ is a minimizing sequence for the reduced functional $E_{J}^{\mu}$.

(3) The functional $E$ has a minimum over $\Gamma_{M, J}^{\mu}$ if and only if the functional $E_{J}^{\mu}$ has a minimum over the constraint set $E_{M}^{\mu}$. In that case the corresponding minimizers $f(\cdot, \cdot)$ and $\rho(\cdot)$ also verify $\rho(\cdot)=\int f(\cdot, v) d v$.

For a proof cf. 77. The aim of this paper is to show how the analysis of the reduced problem again fits into the general framework of the result of A. Burchard and Y. Guo. Before stating the main theorem, we introduce the following definition:

Definition 1.2. Let $n \in \mathbb{N}$. A mapping $T$ is called a translation if there exists a shift vector $a \in \mathbb{R}^{n}$ such that $T f(\cdot)=f(\cdot-a)$, for any function $f: \mathbb{R}^{n} \rightarrow \mathbb{R}$.

We shall prove the following theorem:

Theorem 1.3. Let $\left(\rho_{n}\right) \subset F_{M}^{\mu}$ be a minimizing sequence of the functional $E_{J}^{\mu}$ and let $\mu \in] 0,7 / 2\left[\right.$. Then there exists a sequence of translations $T_{n}$, a subsequence of $\left(\rho_{n}\right)$ (which we denote again by $\left.\left(\rho_{n}\right)\right)$, and $R>0$ such that

$$
\begin{aligned}
& \int_{|x| \geq R} T_{n} \rho_{n}(x) d x \rightarrow 0 \quad \text { as } \quad n \rightarrow \infty, \\
& T_{n} \rho_{n} \rightarrow \rho_{0} \quad \text { strongly } \quad \text { in } \quad L^{\frac{2 \mu+5}{2 \mu+3}}\left(\mathbb{R}^{3}\right),
\end{aligned}
$$


and

In addition to this,

$$
\int_{B_{R}} \rho_{0}(x) d x=M \quad \text { and } \quad \operatorname{supp}\left(\rho_{0}\right) \subset B_{R}
$$

$$
\nabla U_{T_{n} \rho_{n}} \rightarrow \nabla U_{\rho_{0}} \quad \text { strongly in } L^{2}\left(\mathbb{R}^{3}\right) \quad \text { as } \quad n \rightarrow \infty,
$$

and $\rho_{0}$ is a minimizer of the functional $E_{J}^{\mu}$ over the set $F_{M}^{\mu}$.

In $[7$ the authors used a concentration-compactness type argument in the spirit of $[\underline{6}$, but here we give a different proof.

2. Proof of the main result. The crucial part of the proof is to carefully examine the behavior of the spherically symmetric minimizing sequences and then apply [1, Thm. 1]. In order to emphasize the general nature of this method we follow the setup provided in [5], where the author analyzed the problem of minimizing (1.4):

Step1: Concentration implies compactness. The following lemma will be used to treat the behavior of the potential energy along the spherically symmetric minimizing sequences.

Lemma 2.1. Fix any $0<n<5$ and let $\left(\rho_{j}\right) \subset L_{+}^{1+1 / n}\left(\mathbb{R}^{3}, M\right)$ be a sequence of functions such that $\rho_{j} \rightarrow \rho_{0}$ weakly in $L^{1+1 / n}\left(\mathbb{R}^{3}\right)$. Assume that

$$
\lim _{j \rightarrow \infty} \int_{|x| \geq R} \rho_{j}=0
$$

for some $R>0$, i.e., the mass remains asymptotically concentrated in the ball of radius $R$. Then

$$
E_{\text {pot }}\left(\rho_{j}-\rho_{0}\right) \rightarrow 0, \quad j \rightarrow \infty .
$$

Proof. Let us set $\sigma_{j}:=\rho_{j}-\rho_{0}$. For $\delta>0$ let us split the integral

$$
I_{j}:=E_{\mathrm{pot}}\left(\sigma_{j}\right)=-\int \frac{\sigma_{j}(x) \sigma_{j}(y)}{|x-y|} d x d y
$$

into three parts,

$$
I_{j}=I_{j, 1}+I_{j, 2}+I_{j, 3},
$$

where

$$
\begin{gathered}
|x-y|<\delta \text { for } I_{j, 1}, \quad|x-y| \geq \delta \wedge(|x| \geq R \vee|y| \geq R) \text { for } I_{j, 2}, \\
|x-y| \geq \delta \wedge|x|<R \wedge|y|<R \text { for } I_{j, 3} .
\end{gathered}
$$

Obviously, $\int \sigma_{j} d x \leq 2 M$ for every $j$. Since $2 n /(n+1)+2 /(n+1)=2$, we get by Young's inequality

$$
\left|I_{j, 1}\right| \leq C|| \sigma_{j} \|_{1+1 / n}^{2}|| \mathbf{1}_{B_{\delta}}|.|^{-1}||_{n+1 / 2} \leq C\left(\int_{0}^{\delta} r^{\frac{3-n}{2}} d r\right)^{2 /(n+1)} \rightarrow 0
$$

if $\delta \rightarrow 0$, uniformly in $j$ (note that $\mathbf{1}_{A}$ stands for the characteristic function of the set $A$ and $B_{r}$ refers to the ball of radius $r$, for $r>0$ ). Furthermore,

$$
\left|I_{j, 2}\right| \leq \frac{2 M}{\delta} \int_{|x| \geq R}\left|\sigma_{j}(x)\right| d x \rightarrow 0,
$$


as $j \rightarrow \infty$, for any fixed $\delta$. Finally by Hölder's inequality

$$
\left|I_{j, 3}\right|=\left|\int \sigma_{j}(x) h_{j}(x)\right| \leq\left.\left\|\sigma_{j}\right\|_{L^{1+1 / n}\left(\mathbb{R}^{3}\right)}|| h\right|_{L^{1+n}\left(\mathbb{R}^{3}\right)},
$$

where, in a pointwise sense

$$
h_{j}(x):=\mathbf{1}_{B_{R}}(x) \int_{|x-y| \geq \delta} \mathbf{1}_{B_{R}}(y) \frac{\sigma_{j}(y)}{|x-y|} d y \rightarrow 0
$$

which follows by the weak convergence of $\sigma_{j}$ and the fact that we are integrating $\sigma_{j}$ against a test function in $L^{1+n}$. But, since $h_{j} \leq \frac{2 M}{\delta}$ for every $j$, we conclude by Lebesgue's dominated convergence theorem that $h_{j} \rightarrow 0$ in $L^{1+n}$ and thus $\left|I_{j, 3}\right| \rightarrow 0$ as $j \rightarrow \infty$. The lemma is proven.

Step 2: Behavior under rescaling. In analogy to [5] (Section 5, Step 3) one needs to examine the behavior of the involved functional under scaling. The statement and proof can be found in [7. For the sake of completeness we state the result.

Lemma 2.2. The infima $I_{M, J}^{\mu}$ and $R_{M, J}^{\mu}$ verify:

(1) $I_{M, J}^{\mu}=R_{M, J}^{\mu}=M^{\frac{7-2 \mu}{3}} J^{\frac{2(\mu+1)}{3}} I_{1,1}^{\mu}$,

(2) $-\infty<I_{M, J}^{\mu}=R_{M, J}^{\mu}<0$.

Corollary 1. Any minimizing sequence of $E_{J}^{\mu}$ over $F_{M}^{\mu}$ is uniformly bounded in $L^{\frac{2 \mu+5}{2 \mu+3}}\left(\mathbb{R}^{3}\right)$.

Proof. Let $\left(\rho_{n}\right)$ be a minimizing sequence. From the proof of Lemma 2.2 (cf. [7]) it is then easy to conclude that every minimizing sequence is uniformly bounded in $L^{\frac{6}{5}}\left(\mathbb{R}^{3}\right)$ and that $\left(E_{\text {pot }}\left(\rho_{n}\right)\right)$ is also uniformly bounded. Finally, from the definition of $E_{J}^{\mu}$ we deduce the claim.

Step 3: Spherically symmetric minimizing sequences remain concentrated. Now we state the crucial concentration argument for spherically symmetric minimizing sequences of the reduced problem.

LEMMA 2.3. Let us define

$$
R_{0}:=\frac{M^{2}}{-k R_{J, M}^{\mu}} \quad \text { where } \quad k:=7 / 3-2 \mu / 3 .
$$

Let $\rho \in F_{M}^{\mu}$ be spherically symmetric, $R^{\prime}>0$ and define

$$
m:=\int_{|x| \geq R^{\prime}} \rho(x) d x .
$$

Then the following inequality holds:

$$
E_{J}^{\mu}(\rho) \geq R_{J, M}^{\mu}+m(M-m)\left[\frac{1}{R_{0}}-\frac{1}{R^{\prime}}\right] .
$$

If $R^{\prime}>R_{0}$, then for every spherically symmetric minimizing sequence $\left(\rho_{n}\right) \subset F_{M}^{\mu}$ of the functional $E_{J}^{\mu}$ we have

$$
\lim _{n \rightarrow \infty} \int_{|x| \geq R^{\prime}} \rho_{n}(x) d x=0 .
$$


Proof. Although the statement of this lemma is completely analogous to Step 4, Section 5 of [5], the proof is based on somewhat more complicated arguments, due to the more complicated nature of the scaling relations in Lemma 2.2. We define $\rho_{1}:=\mathbf{1}_{B_{R^{\prime}}} \rho$ and $\rho_{2}:=\rho-\rho_{1}$, and also

$$
\alpha_{1}:=\frac{\int \rho_{1}^{\frac{2 \mu+5}{2 \mu+3}} d x}{\int \rho^{\frac{2 \mu+5}{2 \mu+3}} d x} \text { and } \alpha_{2}:=\frac{\int \rho_{2}^{\frac{2 \mu+5}{2 \mu+3}} d x}{\int \rho^{\frac{2 \mu+5}{2 \mu+3}} d x} .
$$

By keeping in mind that

$$
K=\frac{K_{1,1}}{J^{\frac{2(\mu+1)}{3}}}
$$

we obtain

$$
\begin{aligned}
& E_{J}^{\mu}(\rho)=E_{\alpha_{1} \frac{\mu}{\mu+1} J}^{\mu}\left(\rho_{1}\right)+E_{\alpha_{2} \frac{\mu}{\mu+1} J}^{\mu}\left(\rho_{2}\right)-\iint \frac{\rho_{1}(x) \rho_{2}(y)}{|x-y|} d x d y \\
& \geq R_{m, \alpha_{1} \frac{\mu}{\mu+1} J}^{\mu}\left(\rho_{1}\right)+R_{M-m, \alpha_{2} \frac{\mu}{\mu+1} J}^{\mu}\left(\rho_{2}\right)-\frac{m(M-m)}{R^{\prime}} \\
& =M^{\frac{7-2 \mu}{3}} J^{\frac{2(\mu+1)}{3}} R_{1,1}^{\mu}\left(\alpha_{1}^{\frac{2 \mu}{3}}\left(\frac{m}{M}\right)^{\frac{7-2 \mu}{3}}+\alpha_{2} \frac{2 \mu}{3}\left(\frac{M-m}{M}\right)^{\frac{7-2 \mu}{3}}\right) \\
& -\frac{m(M-m)}{R^{\prime}} \\
& =R_{M, J}^{\mu}\left(\left(\left(\frac{m}{M}\right)^{\frac{7}{3}}\right)^{\frac{7-2 \mu}{7}}\left(\alpha_{1}^{\frac{7}{3}}\right)^{\frac{2 \mu}{7}}+\left(\left(\frac{M-m}{M}\right)^{\frac{7}{3}}\right)^{\frac{7-2 \mu}{7}}\left(\alpha_{2}{ }^{\frac{7}{3}}\right)^{\frac{2 \mu}{7}}\right) \\
& -\frac{m(M-m)}{R^{\prime}} \\
& \geq R_{M, J}^{\mu}\left[\alpha_{1}^{\frac{7}{3}}+\alpha_{2}^{\frac{7}{3}}\right]^{\frac{2 \mu}{7}}\left[\left(\frac{m}{M}\right)^{\frac{7}{3}}+\left(\frac{M-m}{M}\right)^{\frac{7}{3}}\right]^{\frac{7-2 \mu}{7}}-\frac{m(M-m)}{R^{\prime}} \\
& \geq R_{M, J}^{\mu}\left[\alpha_{1}+\alpha_{2}\right]^{\frac{2 \mu}{7}}\left[\left(\frac{m}{M}\right)^{\frac{7}{3}}+\left(\frac{M-m}{M}\right)^{\frac{7}{3}}\right]^{\frac{7-2 \mu}{7}}-\frac{m(M-m)}{R^{\prime}} \\
& =R_{M, J}^{\mu}\left[\left(\frac{m}{M}\right)^{\frac{7}{3}}+\left(\frac{M-m}{M}\right)^{\frac{7}{3}}\right]^{\frac{7-2 \mu}{7}}-\frac{m(M-m)}{R^{\prime}} \\
& \geq R_{M, J}^{\mu}\left[1-\frac{7}{3} \frac{M-m}{M} \frac{m}{M}\right]^{\frac{7-2 \mu}{7}}-\frac{m(M-m)}{R^{\prime}}
\end{aligned}
$$

where we used the scaling relations from Lemma 2.2, the fact that $R_{M, J}^{\mu}$ is negative, $\alpha_{1}+\alpha_{2}=1$, Newton's theorem for spherically symmetric potentials (cf. [4]), the discrete Hölder's inequality, and the fact that for $x \in[0,1]$ we have

$$
x^{\frac{7}{3}}+(1-x)^{\frac{7}{3}} \leq 1-\frac{7}{3} x(1-x) .
$$

For any $a, b>0$ and $0<\alpha<1$ the following inequality ([3, Thm. 41]) holds:

$$
b^{\alpha}-a^{\alpha} \geq \alpha b^{\alpha-1}(b-a)
$$


By combining it with the previous estimates we obtain:

$$
\begin{aligned}
E_{J}^{\mu}(\rho)-R_{M, J}^{\mu} & \geq-R_{M, J}^{\mu}\left(1-\left[1-\frac{7}{3} \frac{M-m}{M} \frac{m}{M}\right]^{\frac{7-2 \mu}{7}}\right)-\frac{m(M-m)}{R^{\prime}} \\
& \geq-\frac{7-2 \mu}{3} \frac{M-m}{M} \frac{m}{M} R_{M, J}^{\mu}-\frac{m(M-m)}{R^{\prime}} \\
& =m(M-m)\left[\frac{1}{R_{0}}-\frac{1}{R^{\prime}}\right]
\end{aligned}
$$

which proves the first claim of the lemma. The concentration property is now a corollary of the first claim, and it is proven by a contradiction argument, in exactly the same way as it was done in Step 4, Section 5 of [5].

Step 4: Removing the symmetry assumption. Let $\left(\rho_{n}\right) \subset F_{M}^{\mu}$ be a minimizing sequence of the functional $E_{J}^{\mu}$. Then the the sequence of spherically symmetric rearrangements $\left(\rho_{n}^{*}\right)$ is also a minimizing sequence. According to Corollary 1, we conclude that $\left(\rho_{n}^{*}\right)$ is uniformly bounded in $L^{\frac{2 \mu+5}{2 \mu+3}}\left(\mathbb{R}^{3}\right)$ and by the theorem of Banach-Alaoglu, we conclude that there exists a subsequence of $\left(\rho_{n}^{*}\right)$, still denoted by $\left(\rho_{n}^{*}\right)$, such that

$$
\rho_{n}^{*} \rightarrow \rho^{\prime} \quad \text { weakly in } \quad L^{\frac{2 \mu+5}{2 \mu+3}}\left(\mathbb{R}^{3}\right)
$$

for some $\rho^{\prime} \in L^{\frac{2 \mu+5}{2 \mu+3}}\left(\mathbb{R}^{3}\right)$. Because of Lemma 2.3 we know that

$$
\lim _{n \rightarrow \infty} \int_{|x| \geq R_{0}} \rho_{n}^{*}(x) d x=0, \quad n \in \mathbb{N},
$$

where we choose $R_{0}$ like in Lemma 2.3. This fact combined with the weak convergence of $\left(\rho_{n}^{*}\right)$ easily implies

$$
\operatorname{supp}\left(\rho^{\prime}\right) \subset B_{R_{0}}, \quad \int \rho^{\prime} d x=M
$$

Lemma 2.1 now implies

$$
\lim _{n \rightarrow \infty} E_{\text {pot }}\left(\rho_{n}^{*}-\rho^{\prime}\right)=0 .
$$

By convexity of $\Psi$ and by Mazur's lemma it is easy to deduce that

$$
\left(\int \Psi\left(\rho^{\prime}\right) d x\right)^{\frac{2 \mu+3}{3}} \leq \limsup _{n \rightarrow \infty}\left(\int \Psi\left(\rho_{n}^{*}\right) d x\right)^{\frac{2 \mu+3}{3}}
$$

(cf. 2] or [5]). This implies immediately that $\rho^{\prime}$ is a minimizer and hence

$$
\left(\int \Psi\left(\rho_{n}^{*}\right) d x\right)^{\frac{2 \mu+3}{3}} \rightarrow\left(\int \Psi\left(\rho^{\prime}\right) d x\right)^{\frac{2 \mu+3}{3}} .
$$

Moreover,

$$
\begin{aligned}
E_{\mathrm{pot}}\left(\rho_{n}\right) & =E_{J}^{\mu}\left(\rho_{n}\right)-\left(\int \Psi\left(\rho_{n}\right) d x\right)^{\frac{2 \mu+3}{3}}=E_{J}^{\mu}\left(\rho_{n}\right)-\left(\int \Psi\left(\rho_{n}^{*}\right) d x\right)^{\frac{2 \mu+3}{3}} \\
& \left.\rightarrow E_{J}^{\mu}\left(\rho^{\prime}\right)\right)-\left(\int \Psi\left(\rho^{\prime}\right) d x\right)^{\frac{2 \mu+3}{3}}=E_{\mathrm{pot}}\left(\rho^{\prime}\right) .
\end{aligned}
$$

We now apply ([1, Thm. 1]) to conclude that there exists a sequence of translations $T_{n}^{\prime}$ such that

$$
\lim _{n \rightarrow \infty}\left\|\nabla U_{T_{n}^{\prime} \rho_{n}}-\nabla U_{\rho^{\prime}}\right\|_{2}=0 .
$$


We easily see that $\lim _{n \rightarrow \infty} E_{\text {pot }}\left(\rho_{n}^{*}\right)=\lim _{n \rightarrow \infty} E_{\text {pot }}\left(T_{n}^{\prime} \rho_{n}\right)=E_{\text {pot }}\left(\rho^{\prime}\right)$. Let us now set $R:=3 R_{0}$. Due to Riesz's rearrangement inequality, following the splitting idea from Lemma 3.1 in [1] (Confinement to a ball), we obtain:

$$
\begin{aligned}
2 E_{\mathrm{pot}}\left(\rho_{n}^{*}\right)-2 E_{\mathrm{pot}}\left(\rho_{n}\right) & \geq \iint \rho_{n}^{*}(x) \rho_{n}^{*}(y) \min \left[\frac{1}{|x-y|}, \frac{1}{2 R_{0}}\right] d x d y \\
& -\iint \rho_{n}(x) \rho_{n}(y) \min \left[\frac{1}{|x-y|}, \frac{1}{2 R_{0}}\right] d x d y \geq 0 .
\end{aligned}
$$

By adding and subtracting the quantity

$$
\iint \rho_{n}^{*}(x) \rho_{n}^{*}(y) \frac{1}{2 R_{0}} d x d y
$$

we get the following:

$$
\begin{aligned}
2 E_{\mathrm{pot}} & \left(\rho_{n}^{*}\right)-2 E_{\mathrm{pot}}\left(\rho_{n}\right) \geq \iint \rho_{n}^{*}(x) \rho_{n}^{*}(y) \frac{1}{2 R_{0}} d x d y \\
& -\iint \rho_{n}(x) \rho_{n}(y) \min \left[\frac{1}{|x-y|}, \frac{1}{2 R_{0}}\right] d x d y \\
& +\iint \rho_{n}^{*}(x) \rho_{n}^{*}(y) \min \left[\frac{1}{|x-y|}, \frac{1}{2 R_{0}}\right] d x d y-\iint \rho_{n}^{*}(x) \rho_{n}^{*}(y) \frac{1}{2 R_{0}} d x d y \\
= & \iint \rho_{n}(x) \rho_{n}(y)\left[\frac{1}{2 R_{0}}-\min \left[\frac{1}{|x-y|}, \frac{1}{2 R_{0}}\right]\right] d x d y \\
& +\iint \rho_{n}^{*}(x) \rho_{n}^{*}(y)\left[\min \left[\frac{1}{|x-y|}, \frac{1}{2 R_{0}}\right]-\frac{1}{2 R_{0}}\right] d x d y \\
= & \iint \rho_{n}(x) \rho_{n}(y)\left[\frac{1}{2 R_{0}}-\min \left[\frac{1}{|x-y|}, \frac{1}{2 R_{0}}\right]\right] d x d y \\
& +\iint_{|x| \geq R_{0} \vee|y| \geq R_{0}} \rho_{n}^{*}(x) \rho_{n}^{*}(y)\left[\min \left[\frac{1}{|x-y|}, \frac{1}{2 R_{0}}\right]-\frac{1}{2 R_{0}}\right] d x d y \\
\geq & {\left[\frac{1}{2 R_{0}}-\frac{1}{R}\right] \iint_{|x-y| \geq R} \rho_{n}(x) \rho_{n}(y) d x d y } \\
+ & \iint_{\left(|x| \geq R_{0} \vee|y| \geq R_{0}\right) \wedge\left(|x-y| \geq 2 R_{0}\right)} \frac{\rho_{n}^{*}(x) \rho_{n}^{*}(y)}{|x-y|} d x d y \\
& -\frac{1}{2 R_{0}} \iint_{\left(|x| \geq R_{0} \vee|y| \geq R_{0}\right) \wedge\left(|x-y| \geq 2 R_{0}\right)} \rho_{n}^{*}(x) \rho_{n}^{*}(y) d x d y \\
= & : A_{n}+B_{n}-C_{n} .
\end{aligned}
$$

Here we used the equimeasurability of the rearrangements. According to the proof of Lemma 3.1 in [1] we conclude that there exists a translation $T_{n}$ such that $A_{n} \geq$ $\left[\frac{1}{2 R_{0}}-\frac{1}{R}\right]\left(\int_{|x| \geq R} T_{n} \rho_{n}(x) d x\right)^{2}$. By letting $n \rightarrow \infty$ it is a direct consequence of (2.1) that both $B_{n}$ and $C_{n}$ tend to 0 as $n \rightarrow \infty$. So we get

$$
\int_{|x| \geq R} T_{n} \rho_{n}(x) d x \rightarrow 0 \quad \text { as } \quad n \rightarrow \infty .
$$


Since $T_{n} \rho_{n}$ is a minimizing sequence, it is uniformly bounded in $L^{\frac{2 \mu+5}{2 \mu+3}}\left(\mathbb{R}^{3}\right)$ which implies that there exists some $\rho_{0}$ such that $T_{n} \rho_{n} \rightarrow \rho_{0}$ weakly in $L^{\frac{2 \mu+5}{2 \mu+3}}\left(\mathbb{R}^{3}\right)$, and $(2.2)$ implies

$$
\int_{B_{R}} \rho_{0} d x=M \quad \text { and } \operatorname{supp}\left(\rho_{0}\right) \subset B_{R} .
$$

Now by Lemma 2.1 we conclude

$$
\nabla U_{T_{n} \rho_{n}} \rightarrow \nabla U_{\rho_{0}} \quad \text { strongly in } L^{2}\left(\mathbb{R}^{3}\right), \quad n \rightarrow \infty,
$$

which, again combined with the convexity of the functional $\Psi(\rho)$, allows for the conclusion that $\rho_{0}$ is a minimizer of our variational problem. Eqn. (2.3) also implies

$$
\lim _{n \rightarrow \infty} \int\left(T_{n} \rho_{n}\right)^{\frac{2 \mu+5}{2 \mu+3}} d x=\int \rho_{0}^{\frac{2 \mu+5}{2 \mu+3}} d x
$$

which means that $\left\|T_{n} \rho_{n}\right\|_{L^{\frac{2 \mu+5}{2 \mu+3}\left(\mathbb{R}^{3}\right)}}$ converges to $\left\|\rho_{0}\right\|_{L^{\frac{2 \mu+5}{2 \mu+3}\left(\mathbb{R}^{3}\right)}}$, and this fact, combined with the weak convergence, implies the strong convergence in the space $L^{\frac{2 \mu+5}{2 \mu+3}}\left(\mathbb{R}^{3}\right)$. This completes the proof of Theorem 1.3 .

\section{REFERENCES}

[1] Burchard, A., Y. Guo: Compactness via Symmetrization. J. Functional Analysis, 214, 40-73, 2004. MR 2079885 (2005g:46061)

[2] Hadžić, M.: Compactness and stability of some systems of nonlinear PDE-s in galactic dynamics. Diploma thesis, University of Vienna, 2005.

[3] Hardy, G., J. E. Littlewood, G. Polya: Inequalities. Cambridge Mathematical Press, Cambridge 1952, 2nd edition. MR0046395 (13:727e)

[4] Lieb, E. H., M. Loss: Analysis. American Math. Soc., Providence, 2001. MR1817225|(2001i:00001)

[5] Rein, G.: Nonlinear Stability of Newtonian Galaxies and Stars from a Mathematical Perspective, Nonlinear Dynamics in Astronomy and Physics. Annals of the New York Academy of Sciences, 1045, 103-119, 2005.

[6] Rein, G.: Reduction and a concentration-compactness principle for energy-Casimir functionals. SIAM J. on Math. Anal., 33, 896-912, 2002. MR.1884728 (2003a:35006)

[7] Sánchez, Ó., J. Soler: Orbital stability for polytropic galaxies. Annales de l'Institut Henri Poincare, "Analyse non lineaire". To appear. 